\title{
Combination Therapy Using Gemcitabine and Radioimmunotherapy in Nude Mice with Small Peritoneal Metastases of Colonic Origin
}

\author{
Manuel J. Koppe, ${ }^{1,2}$ Wim J. G. Oyen, ${ }^{2}$ Robert P. Bleichrodt, ${ }^{1}$ Albert A. Verhofstad, ${ }^{3}$ \\ David M. Goldenberg, ${ }^{4}$ and Otto C. Boerman ${ }^{2}$ \\ Departments of ${ }^{1}$ Surgery, ${ }^{2}$ Nuclear Medicine, and ${ }^{3}$ Pathology, Radboud University Nijmegen Medical \\ Center, Nijmegen, The Netherlands. \\ ${ }^{4}$ The Garden State Cancer Center, Center for Molecular Medicine and Immunology, Belleville, NJ
}

\begin{abstract}
Introduction: Gemcitabine has been shown to exert a radiosensitizing effect in various epithelial cancers. The aim of the present studies was to investigate whether the efficacy of radioimmunotherapy (RIT) using the ${ }^{131}$ I-labeled anti-CEA monoclonal antibody (MAb) MN-14 could be enhanced by coadministration of gemcitabine in nude mice with small $(1-3 \mathrm{~mm})$ peritoneal metastases of colonic origin. Materials and methods: Firstly, the maximum tolerated dose (MTD) of gemcitabine was determined, when administered intraperitoneally at two different dosing schedules $(0.11-3.0 \mathrm{mg} / \mathrm{mouse} /$ administration on days 0 , 3, 6, and 9, or 0.022-0.60 mg/mouse/administration on days $0,1,2,3$, and 4). In two separate therapy studies in which these two administration regimens were applied, the efficacy of gemcitabine monotherapy was compared to that of RIT alone $\left(125 \mu \mathrm{Ci}{ }^{131} I-M N-14 /\right.$ mouse) or RIT combined with gemcitabine. Results: When administered every 3rd day for a total of 4 administrations, or daily for 5 consecutive days, the gemcitabine was considered safe at $0.33 \mathrm{mg} / \mathrm{mouse} / \mathrm{administration}$ and $0.066 \mathrm{mg} / \mathrm{mouse} / \mathrm{administra-}$ tion, respectively. In the first therapy study, median survival of the control mice was 39 days. Gemcitabine monotherapy at $0.11 \mathrm{mg}$ or $0.33 \mathrm{mg} / \mathrm{mouse} /$ administration every $3 \mathrm{rd}$ day (total, 4 administrations) resulted in a median survival of 52 and 57 days, respectively $(p=0.0003$, compared to controls). RIT alone resulted in a median survival of 66 days ( $p<0.0001$, compared to controls). The combination of RIT and gemcitabine coadministration resulted in a median survival of 73 and 94 days, respectively $(p=0.12$, for trend). In the second therapy study, median survival of the control mice was 48 days, which was similar to the median survival of the mice treated with daily administrations of gemcitabine monotherapy at 0.022 $\mathrm{mg} / \mathrm{mouse} /$ administration on 5 consecutive days (49 days; $p=0.17$ ). RIT alone resulted in a significantly improved median survival of 66 days ( $p==0.0010$, compared to controls). Combination therapy using RIT and gemcitabine resulted in a median survival of 64 days, which did not differ significantly from the survival of the mice treated with RIT alone $(p=0.43)$. Conclusions: At the dose regimens employed, gemcitabine did not enhance the efficacy of RIT of experimental small-volume peritoneal carcinomatosis of colonic origin.
\end{abstract}

Key words: radioimmunotherapy, gemcitabine, peritoneal metastases, colon carcinoma

Address reprint requests to: Manuel J. Koppe; Department of Surgery, Radboud University Nijmegen Medical Center; P.O. Box 9101, 6500 HB Nijmegen, The Netherlands; Tel.: +31-24-3616421; Fax: +31-24-3540501

E-mail: m.koppe@chir.umcn.nl 


\section{INTRODUCTION}

Despite the promising results of radioimmunotherapy (RIT) using radiolabeled monoclonal antibodies (MAbs) for the treatment of nonHodgkin's lymphoma (NHL), the therapeutic efficacy of RIT in solid tumors has been modest at best. Solid cancers are targeted less efficiently with radiolabeled antibodies than hematological malignancies, which has been attributed to the presence of various physiological barriers between the circulation and the tumor cell surface. It has been pointed out that the vascular endothelium, the relatively large transport distances in the tissue, and the enhanced interstitial pressure in the tumor tissue hamper the penetration of antibodies into the tumor tissue to bind to their target antigen. ${ }^{1}$ As a result, tumor uptake of radiolabeled MAbs and, consequently, the radiation doses delivered to solid lesions are, in most cases, too low to induce objective tumor responses. Several innovative approaches have been pursued with the aim to improve the efficacy of RIT. These include strategies to improve the localization and retention of radiolabeled MAbs in the tumor (e.g., using high-affinity MAbs²), strategies to accelerate the blood clearance of the radiolabel (e.g., using antibody fragments ${ }^{3}$ or the pretargeting method ${ }^{4}$ ), and strategies to increase the sensitivity of the tumor cells to radiation (e.g., using radiosensitizers ${ }^{5-7}$ ).

Gemcitabine ( $2^{\prime}$-deoxy-2' $2^{\prime}$-difluorocytidinemonohydrochloride) is a pyrimidine analog and has been shown to exert antitumor effects in a wide range of solid tumors, as well as some hematological malignancies. ${ }^{8}$ Gemcitabine acts by depleting the deoxynucleoside triphosphate pool and is incorporated in the DNA, in the same way as 5-fluorouracil (5-FU), thereby inhibiting DNA synthesis and, probably, DNA repair. ${ }^{9}$ Gemcitabine has been shown to sensitize a wide range of mainly epithelial cancers to radiation, including adenocarcinoma of the pancreas and colon, and squamous-cell carcinoma. ${ }^{10}$ Although in most chemoradiotherapy studies gemcitabine was combined with external beam radiation, in recent years, a few studies have been published reporting radiosensitizing effects of gemcitabine when combined with RIT in animal models of pancreatic cancer and colon cancer. ${ }^{5-7,11}$

We have previously characterized a nude mouse model for RIT of small-volume peritoneal carcinomatosis of colorectal origin using the human colon carcinoma cell line, LS174T. ${ }^{12}$ RIT using the radiolabeled anti-CEA MAb MN-14 proved very effective in inhibiting the growth of intraperitoneally (i.p.) growing tumor nodules, even at relatively low activity doses. ${ }^{131} \mathrm{I}$ and ${ }^{177} \mathrm{Lu}$ proved to be the most effective radionuclides in this model. ${ }^{13}$ Because gemcitabine has previously been shown to exert some antitumor activity against LS174T ${ }^{14}$ and improve the efficacy of RIT in a subcutaneous (s.c.) tumor model using LS174T, ${ }^{11}$ we hypothesized that gemcitabine coadministration might sensitize the intraperitoneally growing LS174T xenografts to radiation and improve the therapeutic efficacy of RIT in our model of small-volume peritoneal carcinomatosis of colorectal origin. To test this hypothesis, first the maximum tolerated dose (MTD) of gemcitabine was determined in two different administration regimens. Subsequently, the effect of both administration regimens of gemcitabine on the efficacy of RIT, using ${ }^{131} \mathrm{I}$ labeled MN-14, was assessed.

\section{MATERIALS AND METHODS}

\section{Animal Model of Small Peritoneal Metastases}

Male nude BALB/c mice (Charles River Laboratories, Sulzfeld, Germany), 8-10 weeks old and weighing 20-25 g, were used in the experiments. Mice were accustomed to laboratory conditions for at least 1 week before experimental use and were housed under nonsterile standard conditions (temperature $20-24^{\circ} \mathrm{C}$; relative humidity $50 \%-$ $60 \% ; 12$ hours light/12 hours dark) in filtertopped cages (up to 5 mice per cage), on sawdust with free access to animal chow (Snif Voer; Soest, The Netherlands) and water. At the start of therapy, all mice were housed on iron grate floors. Peritoneal metastases were induced, as described previously. ${ }^{12}$ In brief, mice were inoculated i.p. with $1.0 \times 10^{6}$ LS174T cells (CCL 188; American Type Culture Collection, Rockville, MD), suspended in $500 \mu \mathrm{L}$ of RPMI-1640 medium in a 2.5 -mL syringe using a 23 -gauge needle. In this model, the first macroscopic tumor nodules are seen 7-10 days thereafter, whereas bulky peritoneal carcinomatosis develops 3-5 weeks after tumor cell inoculation. All experiments were conducted in accordance with the principles laid out by the revised Dutch Act on Animal Experimentation (1997) and approved by the institutional Animal Welfare Committee 
of the Radboud University Nijmegen (Nijmegen, The Netherlands).

\section{Monoclonal Antibody}

The murine MN-14 MAb is a high-affinity $\left(K_{\mathrm{a}}=\right.$ $10^{9} \mathrm{M}^{-1}$ ) class III anti-CEA IgG $_{1}$ antibody, produced by a hybridoma cell-line culture, kindly provided by Immunomedics, Inc. (Morris Plains, New Jersey). The antibodies were purified by protein-A chromatography, as described previously. ${ }^{15}$ Purity was checked by fast protein liquid chromatography (FPLC) on a Phenomenex (Torrance, CA) Biosep 3000 column (size $300 \times$ $7.8 \mathrm{~mm}$ ), eluted with phosphate-buffered saline (PBS; pH 7.2, $1 \mathrm{~mL} / \mathrm{min}$ ).

\section{Radioiodination}

Antibodies were radioiodinated with ${ }^{131}$ I (MDS Nordion, Fleurus, Belgium, respectively), using the IODOGEN method (1,3,4,6-tetrachloro$3 \alpha, 6 \alpha$-diphenyl-glycoluril; Pierce, Rockford, IL). Briefly, antibodies and ${ }^{131}$ I were incubated at room temperature in $85 \mu \mathrm{L}$ of PBS $(0.10 \mathrm{M}$, $\mathrm{pH}$ 7.4) in a glass vial, coated with $100 \mu \mathrm{g}$ iodogen. The reaction mixture was subsequently separated on a PD-10 column (Amersham Biosciences; Uppsala, Sweden) and eluted with PBS, $0.5 \%$ bovine serum albumin (BSA). Labeling efficiency of the radioiodination reactions was approximately $70 \%$. Specific activities of the ${ }^{131} \mathrm{I}-$ $\mathrm{MN}-14$ preparations were $1.6 \mathrm{MBq} / \mu \mathrm{g}$ (43 $\mu \mathrm{Ci} / \mu \mathrm{g})$ and $0.64 \mathrm{MBq} / \mu \mathrm{g}(17 \mu \mathrm{Ci} / \mu \mathrm{g})$, respectively. In a previous study, we demonstrated that the uptake of the radiolabeled MN-14 antibody in tumor was optimal at $\mathrm{MN}-14$ protein doses up to $25 \mu \mathrm{g} .{ }^{12}$ Therefore, in this study, unlabeled $\mathrm{MN}-14$ was added to the radiolabeled antibody preparations to adjust the total antibody protein dose to $20 \mu \mathrm{g}$ per mouse.

\section{Quality Control of the Radiolabeled Antibody Preparations}

The amount of free ${ }^{131}$ I was determined by instant thin-layer chromatography (ITLC), using ITLC silica gel strips (Gelman Sciences, Inc.; Ann Arbor, MI), with $0.1 \mathrm{M}$ citrate buffer, $\mathrm{pH}$ 6.0 , as the mobile phase. Radiochemical purity of all radiolabeled antibody preparations used in the studies exceeded $97 \%$. The immunoreactive fraction (IRF) of the radiolabeled $\mathrm{MN}-14$ preparations was determined on freshly trypsinized LS174T cells, essentially as described by Lindmo et al. ${ }^{16}$ with minor modifications. Briefly, a fixed amount of labeled antibody $(10,000 \mathrm{cpm})$ was incubated with increasing concentrations of LS174T tumor cells $\left(1.2 \times 10^{6}-20 \times 10^{6}\right.$ cells $/ \mathrm{mL}$ ) in $0.5 \mathrm{~mL}$ binding buffer (RPMI medium containing $0.5 \% \mathrm{BSA}$ and $0.05 \% \mathrm{NaN}_{3}$ ). A duplicate of the lowest cell concentration was incubated in the presence of an excess unlabeled antibody to correct for nonspecific binding. After 6 hours of incubation at $37^{\circ} \mathrm{C}$, the cells were washed and activity in the pellet was determined in a well-type gamma counter. The inverse of the tumor cell bound fraction was plotted against the inverse of the cell concentration and the IRF was calculated from the Y-axis intercept. The IRF of the ${ }^{131} \mathrm{I}-\mathrm{MN}-14$ preparations was $82 \%$ and $77 \%$, respectively. The radiolabeled antibody preparations were administered within 2 hours after radiolabeling.

\section{Gemcitabine}

Gemcitabine (2'-deoxy-2'2'-difluorocytidinemonohydrochloride; Gemzar $\left.{ }^{\circledR}\right)$ was purchased from Ely Lilly Company (Houten, The Netherlands) as a powder in a glass vial (200 mg/vial). Immediately before administration, gemcitabine was dissolved in $0.9 \%$ sodium chloride (saline) to the appropriate concentrations.

\section{Studies to Determine the Maximum Tolerated Gemcitabine Dose}

To determine the MTD of gemcitabine, groups of 6-8 mice were injected i.p. with escalating doses of gemcitabine. Two (2) dosing regimens were investigated in 2 separate experiments. In the first regimen, mice were treated on days 0,3 , 6 , and 9 with gemcitabine at $0.11,0.33,1.0$, or $3.0 \mathrm{mg} / \mathrm{mouse} / \mathrm{administration}$. The second dosing regimen consisted of daily intraperitoneal administrations of $0.022,0.066,0.20$, or 0.60 $\mathrm{mg} / \mathrm{mouse} / \mathrm{administration,} \mathrm{which} \mathrm{was} \mathrm{given} \mathrm{daily}$ on 5 consecutive days. Control mice received saline only. Mice were monitored daily, and body weight was measured daily to ensure that body weight loss did not exceed $20 \%$.

\section{Combination Therapy with Radioimmunotherapy and Gemcitabine}

To assess the radiosensitizing effect of gemcitabine, 2 separate therapy experiments were carried out in which each of the 2 gemcitabine dosing regimens described above was combined with RIT. In both experiments, RIT consisted of $125 \mu \mathrm{Ci}^{131} \mathrm{I}-\mathrm{MN}-14 /$ mouse, which was given i.p. 
10 days after tumor cell inoculation (20 $\mu \mathrm{g} /$ mouse). This activity dose represents approximately $25 \%$ of the maximum tolerated activity dose of ${ }^{131}$ I-labeled $\mathrm{IgG}$ antibodies, which has previously been shown to significantly delay the growth of i.p. growing LS174T xenografts in the present model. ${ }^{13}$ The majority of mice still develop peritoneal carcinomatosis only after 9-10 weeks. Thus, we assumed that a relevant radiosensitizing effect of gemcitabine could be demonstrated at this dose level.

In the first therapy study, mice were treated with either RIT alone $\left(125 \mu \mathrm{Ci}{ }^{131} \mathrm{I}-\mathrm{MN}\right.$ 14/mouse, 10 days after tumor cell inoculation), gemcitabine alone (either $0.11 \mathrm{mg}$ or 0.33 $\mathrm{mg} / \mathrm{mouse} / \mathrm{administration}$ on days $10,13,16$, and 19 after tumor cell inoculation), or ${ }^{131} \mathrm{I}$ $\mathrm{MN}-14$ combined with gemcitabine. In the second therapy study, mice were treated with either RIT alone (125 $\mu \mathrm{Ci}^{131} \mathrm{I}-\mathrm{MN}-14 /$ mouse, 10 days after tumor cell inoculation), gemcitabine monotherapy $(0.022 \mathrm{mg} / \mathrm{per} / \mathrm{mouse}$ on days 10 , $11,12,13$, and 14 after tumor cell inoculation), or RIT combined with gemcitabine. In both studies, control mice received unlabeled $\mathrm{MN}$ 14 in PBS, $0.5 \%$ BSA. Furthermore, control mice and the mice that were treated with RIT monotherapy received i.p. injections of saline instead of gemcitabine (10-12 mice per group).

Mice were monitored until the humane endpoint had been reached, as determined by an experienced and independent animal technician, who was blinded to which treatment mice had received. ${ }^{17}$ At the time of the humane endpoint, mice were usually cachectic and drowsy, showing signs of advanced peritoneal carcinomatosis, such as the presence of bloody ascites or bulky i.p. tumor growth, and were expected to die within 1 or 2 days. When the humane endpoint had been reached, mice were euthanized by $\mathrm{O}_{2} / \mathrm{CO}_{2}$-asphyxiation and immediately dissected. At dissection, all macroscopic tumor deposits were meticulously excised and weighed. The experiments were terminated at 134 and 96 days after tumor cell inoculation, when the remaining mice were euthanized and dissected. The abdominal cavity was conscientiously inspected. Liver, spleen, lungs, pancreas, greater omentum, and the diaphragm were removed for routine histopathological staining and immunohistochemical staining using a rabbit-anti-human anti-CEA polyclonal antibody (A 0115; DakoCytomation, Glostrup, Denmark). ${ }^{13}$

\section{Statistical Analysis}

Statistical analysis was performed by means of the GraphPad Prism 4.00 software (GraphPad Software; San Diego, CA). Single comparisons were analyzed using the nonparametric MannWhitney U test. Multiple comparisons were analyzed using the one-way analysis of variance test. Bonferroni correction for multiple testing was applied. In the therapy studies, comparisons between the groups for differences in survival were analyzed using the log-rank test. All tests were two-sided; the level of statistical significance was set at a $\mathrm{P}$-value of $<0.05$.

\section{RESULTS}

\section{Toxicity of Gemcitabine}

To determine the dose of gemcitabine that could be administered safely, mice received i.p. injections of gemcitabine in 2 dosing regimens. In the first experiment, in which 4 injections of gemcitabine were given every 3rd day, 4 of 6 and 1 of 6 mice that were treated at 3.0 and $1.0 \mathrm{mg} / \mathrm{mouse} /$ administration, respectively, died owing to severe gemcitabine-related toxicity during the treatment period. Body weight loss exceeded $20 \%$ and mice suffered from gastrointestinal toxicity, which consisted of diarrhea, and, in some mice, hematologic toxicity, manifesting by the presence of skin petechiae. When administered at 0.33 or $0.11 \mathrm{mg} /$ administration, none of the mice developed diarrhea or skin petechiae. Figure 1A depicts the relative body weight during the study. Whereas control mice showed a gradual increase in body weight, maximum average body weight loss was $2.4 \% \pm 1.4 \%$ in the mice treated at $0.33 \mathrm{mg} /$ administration (2 days after the first administration) and $1.7 \% \pm$ $2.2 \%$ in the mice treated at $0.11 \mathrm{mg} /$ administration ( 1 day after second administration; $p=0.24$ ).

In the second experiment, in which 5 daily injections of gemcitabine were given, all mice treated at 0.60 or $0.20 \mathrm{mg} / \mathrm{administration}$ and 6 of 8 mice treated at $0.066 \mathrm{mg} /$ administration died owing to severe gemcitabine-related toxicity either during or shortly after the treatment period. Daily administration at $0.022 \mathrm{mg} /$ administration did not result in clinically evident toxicity. As shown in Figure 1B, the maximum average body weight loss amounted to $6.4 \% \pm 3.6 \% 7$ days after the first administration, which did not differ significantly from the maximum weight loss of $4.8 \% \pm 3.6 \%$ in the control mice given daily injections of saline $(p=0.19)$. 


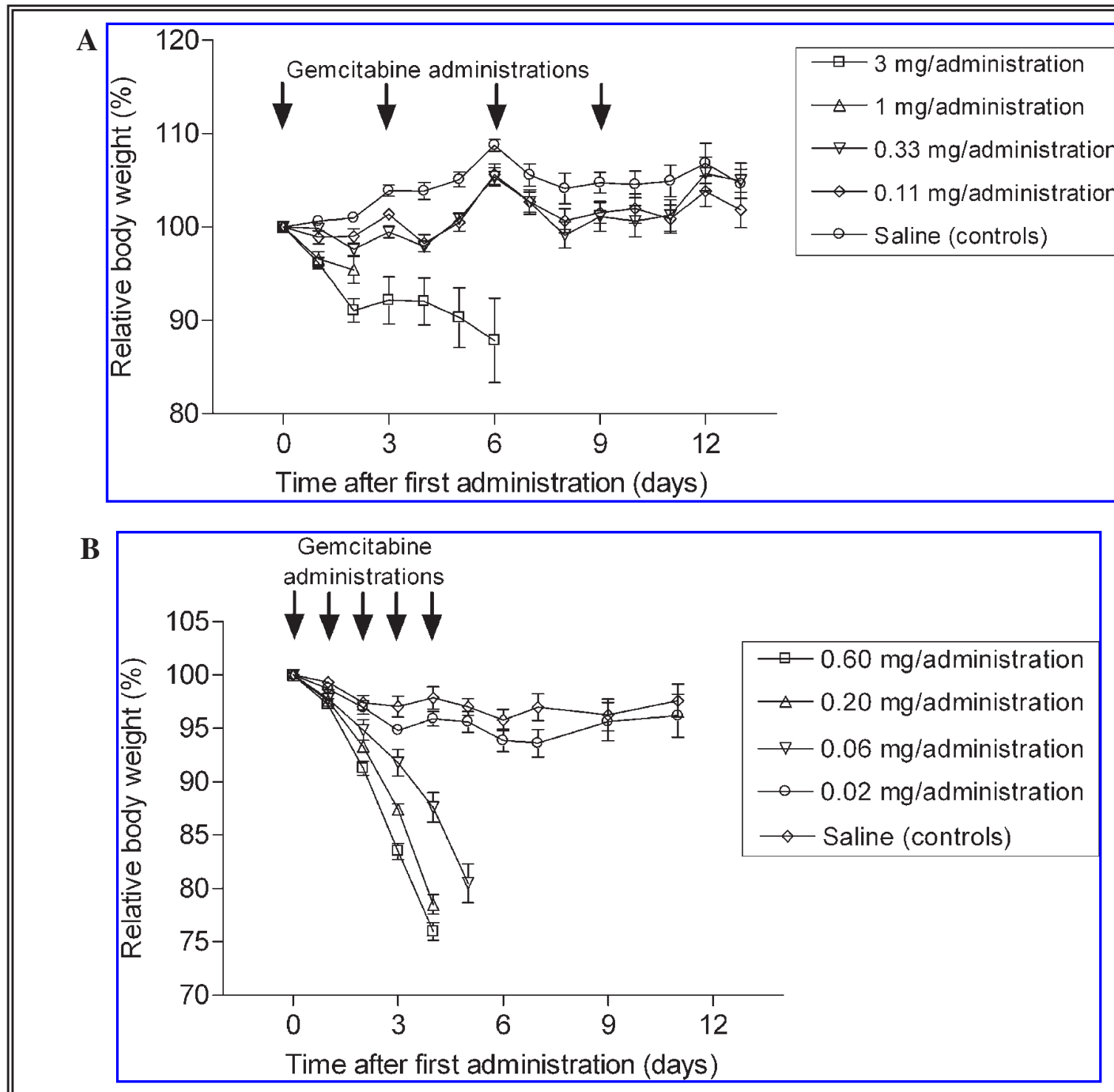

Figure 1. (A) Relative body weight of nude mice after once-daily intraperitoneal (i.p.) administration of gemcitabine at $0,0.11$, 0.33 , 1.0, or $3.0 \mathrm{mg} / \mathrm{administration}$ every 3rd day for a total of 4 administrations. (B) Relative body weight of nude mice after once-daily i.p. administration of gemcitabine at $0,0.022,0.066,0.20$, or $0.60 \mathrm{mg} / \mathrm{administration}$ for a total of 5 administrations.

Based on these observations, gemcitabine was considered safe when administered at 0.33 $\mathrm{mg} / \mathrm{mouse} / \mathrm{administration} \mathrm{every} \mathrm{3rd} \mathrm{day} \mathrm{for} \mathrm{a} \mathrm{to-}$ tal of 4 administrations, or at $0.022 \mathrm{mg} / \mathrm{mouse} / \mathrm{ad}-$ ministration, when administered daily for 5 consecutive days.

\section{RIT Combined with Gemcitabine Administered Every 3rd Day for 4 Consecutive Days}

The survival curves for the various treatment groups are shown in Figure 2A. Median survival of the control mice was 39 days (range, 34-52). Gemcitabine monotherapy at 0.11 or 0.33 $\mathrm{mg} / \mathrm{mouse} /$ administration every 3rd day for a total of 4 administrations resulted in a median survival of 52 days (range, 48-62) and 57 days (range, 52-69), respectively $(p=0.0003$, for trend when compared to controls). RIT alone resulted in a significantly improved median survival of 66 days (range, 55-143; $p<0.0001$, compared to controls). RIT combined with 4 administrations of gemcitabine at 0.11 or 0.33 $\mathrm{mg} / \mathrm{mouse} / \mathrm{administration} \mathrm{every} \mathrm{3rd} \mathrm{day} \mathrm{resulted}$ in a median survival of 73 days (range, 66-101) 


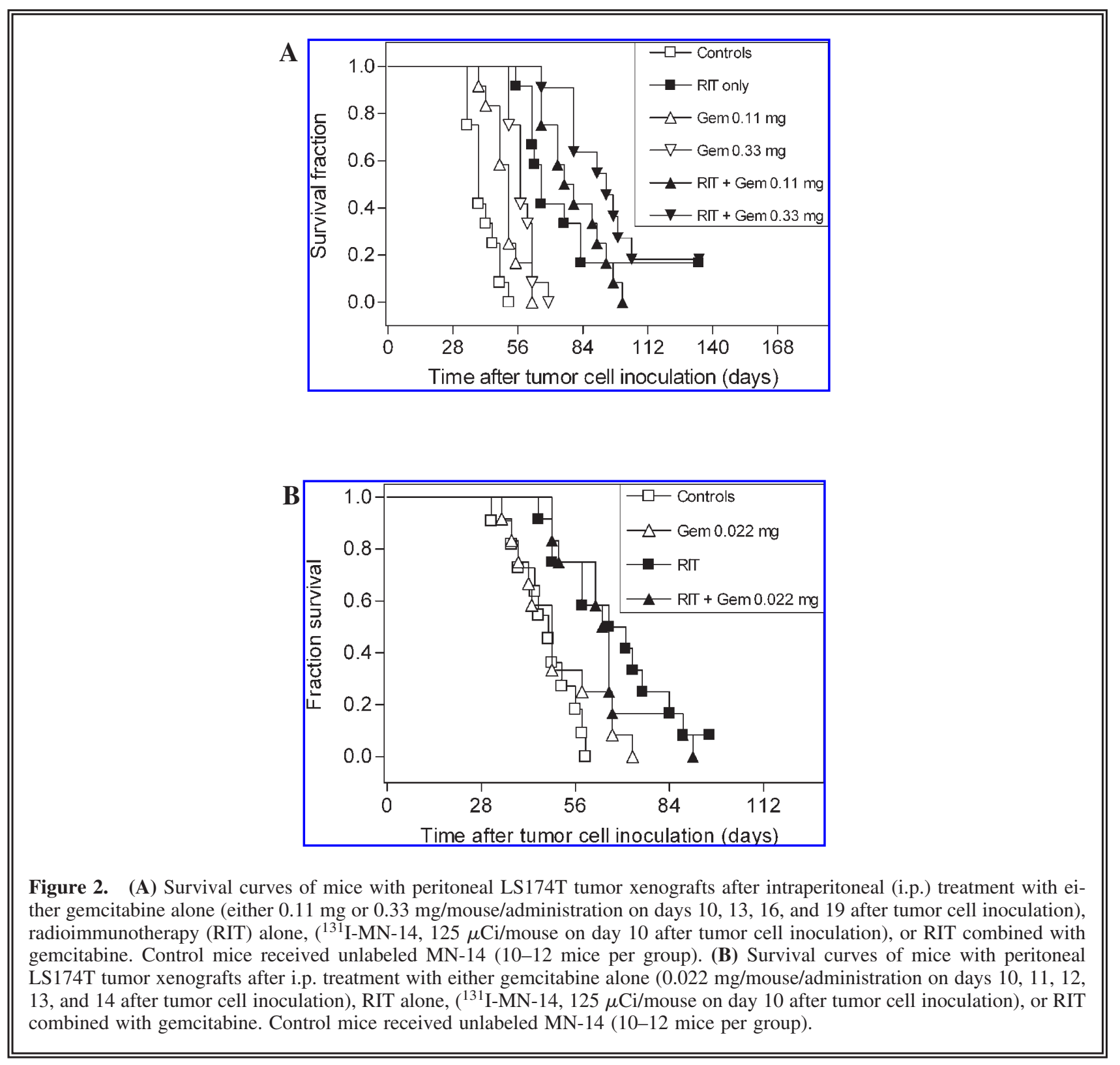

and 94 days (range, 66-143), respectively ( $p=$ 0.12 , for trend compared to RIT alone).

The tumor weight found at dissection at the time mice reached their humane endpoints varied between $4.0 \pm 1.4 \mathrm{~g}$ for the mice treated with gemcitabine monotherapy $0.11 \mathrm{mg} /$ administration and $5.4 \pm 1.1 \mathrm{~g}$ for the mice treated with RIT + gemcitabine $0.33 \mathrm{mg} / \mathrm{administration}(p=$ $0.24)$. The similar tumor mass found in the mice in the various treatment groups demonstrates that there was no bias in determining the humane endpoint. The method of determining and comparing the survival was, therefore, reliable.
At the end of the experiment (143 days after tumor cell inoculation), there were four long-term survivors ( 2 treated with RIT alone; and 2 treated with RIT + gemcitabine $0.33 \mathrm{mg} / \mathrm{mouse} /$ administration), without signs of i.p. tumor growth. At dissection, 2 mice (1 from each group) had macroscopic tumor growth $(0.13$ and $0.06 \mathrm{~g}$, respectively), whereas in the remaining 2 mice there was no evidence of disease. Histopathological examination of relevant organs, including the greater omentum, mesentery, diaphragm, and pancreas, did not reveal residual disease in any of these mice. 


\section{RIT Combined with Gemcitabine Administered Daily for 5 Consecutive Days}

The survival curves for the various treatment groups are shown in Figure 2B. Median survival of the control mice was 48 days (range, 31-58). Daily gemcitabine monotherapy at $0.022 \mathrm{mg} /$ mouse/administration for 5 consecutive days resulted in a median survival of 49 days (range, 34-73; $p=0.17$, compared with controls). RIT alone resulted in a significantly improved median survival of 66 days (range, 45-96; $p=0.0010$, compared with controls). Five (5) daily gemcitabine administrations at $0.022 \mathrm{mg} / \mathrm{mouse} / \mathrm{ad}-$ ministration starting concurrently with RIT resulted in a median survival of 65 days (range, 49-91), which did not differ significantly from the survival of the mice treated with RIT alone $(p=0.43)$.

The tumor weight that was found at dissection at the time mice reached their humane endpoints varied from $4.4 \pm 1.9 \mathrm{~g}$ for the mice treated with RIT + gemcitabine and $6.0 \pm 2.0 \mathrm{~g}$ for the mice treated with gemcitabine monotherapy $(p=$ $0.15)$.

At the end of the experiment (96 days after tumor cell inoculation), 1 mouse treated with RIT only was still alive, without signs of i.p. tumor growth. At dissection, this mouse had no macroscopic tumor growth. Histopathological examination of relevant organs, including the greater omentum, mesentery, diaphragm, and pancreas, did not reveal residual disease.

\section{DISCUSSION}

Radiosensitization can be defined as the use of agents with the aim to increase the sensitivity of tissue to radiation therapy. The primary aim of this study was to investigate the radiosensitizing potential of two gemcitabine treatment schedules when combined with single-dose radioimmunotherapy in a well-characterized animal model of peritoneal carcinomatosis of colorectal origin. Gemcitabine, however, failed to significantly improve the efficacy of radioimmunotherapy in both administration regimens tested.

Originally intended for development as an antiviral agent, gemcitabine was introduced in the late 1980 s as a novel and very potent pyrimidine antimetabolite, with antitumor activity against a wide range of epithelial cancers, including nonsmall-cell- and small-cell lung cancer, breast cancer, pancreatic cancer, head-and-neck squamous- cell cancer, and cervical cancer. ${ }^{18}$ Furthermore, probably because of its interference with DNA repair, gemcitabine has shown to be a potent radiosensitizer. Although only marginally effective when administered as a single agent in patients with advanced colorectal carcinoma, ${ }^{19}$ gemcitabine has been demonstrated to sensitize colorectal carcinoma to radiation therapy in both preclinical and clinical studies. ${ }^{20,21}$ Interestingly, gemcitabine can induce radiosensitization at tissue concentrations 1000 times lower than typical plasma levels obtained with the drug. ${ }^{21}$ Furthermore, the radiosensitizing effects of gemcitabine have been shown to be dose-dependent. ${ }^{18}$ This prompted us to conduct the experimental studies described in this paper.

To date, four reports have been published on the combination of experimental RIT and gemcitabine, three of which are in animal models of pancreatic cancer, ${ }^{5-7}$ and one in an animal model of colon cancer. ${ }^{11}$ The group of Goldenberg published three reports on the efficacy of combination therapy using ${ }^{131}$ I-labeled or ${ }^{90}$ Y-DOTA-labeled chimeric PAM4 MAb and gemcitabine in nude mice bearing s.c. CaPan1 human pancreas carcinoma xenografts. In their first study, mice bearing subcutaneous tumors of approximately 1 $\mathrm{cm}^{3}$ were treated with ${ }^{131}$ I-cPAM4 (100 or 200 $\mu \mathrm{Ci}$ ) alone, gemcitabine alone on days $0,3,6,9$, and 12 at $333 \mathrm{mg} / \mathrm{m}^{2}$ (2 mg/mouse), or the combination of both treatments. ${ }^{5}$ Whereas both monotherapies did not have antitumor effects, the combination of both treatment modalities showed a statistically significant synergistic effect, which led the authors to conclude that gemcitabine lowered the threshold for antitumor response sufficiently, so that the radiation dose provided by the low-dose RIT could still carry antitumor activity. Furthermore, combination therapy was well tolerated, even at the higher RIT activity dose of $200 \mu \mathrm{Ci}$. In their second study, mice with somewhat smaller s.c. tumors $\left(0.5 \mathrm{~cm}^{3}\right)$ were treated with either 3 cycles of $25 \mu \mathrm{Ci}{ }^{90}$ Y-DOTA-labeled cPAM4 only at weeks 0,4 and 7 , or once-weekly i.p. injections of gemcitabine $(6 \mathrm{mg} /$ mouse, which equals $1000 \mathrm{mg} / \mathrm{m}^{2} /$ week), or combination therapy. ${ }^{7}$ Both RIT and gemcitabine monotherapy, when given as monotherapy, had some antitumor effects. When combined, however, there was a supra-additive antitumor effect, at the cost of only minimal toxicity to normal tissues, as evidenced by an average loss in body weight of less than $2 \%$ for all treatment groups. In their last report, RIT using ${ }^{90} \mathrm{Y}$-DOTA-labeled cPAM4 was 
combined with gemcitabine, according to the same dose regimen as utilized in their first study, with ${ }^{131}$ I-cPAM4 in mice bearing $1 \mathrm{~cm}^{3}$ tumors. ${ }^{6}$ Gemcitabine was not effective when given as single treatment but still improved the efficacy of RIT when given in combination with ${ }^{90} \mathrm{Y}$-DOTAcPAM4. Finally, Graves et al. reported on the radiosensitizing effect of gemcitabine when combined with pretargeted RIT in nude mice with human colon cancer xenografts. ${ }^{11}$ In that study, mice bearing 100-200 $\mathrm{mm}^{3}$ s.c. LS174T tumors were treated with either pretargeted RIT, 2 i.p. injections of gemcitabine at $50-200 \mathrm{mg} / \mathrm{kg} 1$ day before and 1 day after administration of ${ }^{131} \mathrm{I}-\mathrm{la}$ beled peptide, or combined modality treatment. Whereas both pretargeted RIT and gemcitabine, given as monotherapies, did not result in significant growth delay of the s.c. tumors, the combination of both treatment modalities resulted in a highly significant delay of tumor growth, which was dependent on the dose of gemcitabine used.

In our study, gemcitabine was very toxic in both dosing regimens tested. The dose levels chosen in both MTD studies were based on the doses utilized in the above-mentioned studies, in which gemcitabine was combined with RIT. Cardillo et al. ${ }^{5}$ and Gold et al. ${ }^{7}$ administered gemcitabine i.p. every 3 rd day for a total of 5 administrations at $2 \mathrm{mg} /$ mouse and reported a maximum mean weight loss of only $4.5 \% \pm 5.5 \%$ on day 7 . In this study, however, 1 of 6 mice treated at 1 $\mathrm{mg} / \mathrm{mouse}$ died of gemcitabine-related toxicity 2 days after the first administration, whereas of the 6 mice treated at $3 \mathrm{mg} / \mathrm{mouse}, 4$ died of acute treatment-related toxicity. Therefore, the MTD of gemcitabine administered every 3rd day for a total of 4 administrations was set at $<1.0$ $\mathrm{mg} /$ mouse. Because once-weekly administrations of up to $6 \mathrm{mg} / \mathrm{mouse}$ have been reported as feasible, ${ }^{6}$ the MTD of gemcitabine, when administered daily for 5 consecutive days, was sought between 0.022 and $0.6 \mathrm{mg} / \mathrm{mouse} /$ administration. Gemcitabine, however, was lethal in the majority of mice treated at $0.066 \mathrm{mg} / \mathrm{administration}$ and in all mice treated at higher doses. Hence, the MTD of daily administrations of gemcitabine on 5 consecutive days was set at only 22 $\mu \mathrm{g} / \mathrm{mouse} / \mathrm{administration}$. The differences between the MTDs found in this study and in those reported by other authors might be attributed to differences in mouse strains.

The most efficacious dosing regimen of gemcitabine to fully exploit its radiosensitizing effect is still a matter of investigation in both preclinical and clinical studies. From preclinical studies on the combination of gemcitabine and external beam radiotherapy (RT), it became clear that the effects of combination therapy become synergistic when gemcitabine treatment precedes RT. ${ }^{21}$ In vitro studies indicated that a brief exposure ( 2 hours) of HT29 colon cancer cells to gemcitabine resulted in radiosensitization for up to 48 hours. $^{22}$ In RIT, the radiation energy is delivered to the tumors over a prolonged period of time. Furthermore, because in a previous study we showed that the uptake of radioiodinated $\mathrm{MN}-14$ in the small i.p. tumor deposits is more than $50 \% \mathrm{ID} / \mathrm{g}$ for up to 72 hours postinjection, ${ }^{12}$ we assumed that, similar to the administration regimen reported by the groups of Cardillo and Gold, repeated administrations of gemcitabine starting concurrently with RIT might effectively sensitize the i.p. tumors to RIT. In contrast to the synergistic effects of gemcitabine when combined with RIT, as reported by other authors, gemcitabine did not enhance the efficacy of RIT in our studies. When administered every 3rd day for a total of 4 administrations, gemcitabine monotherapy still had a modest, albeit significant, antitumor effect, which was dose-dependent. When combined with RIT at this dosing schedule, median survival of the combination therapy groups improved relative to the RIT monotherapy group, although the differences did not reach statistical significance. In the second therapy study, daily administrations of gemcitabine at $0.022 \mathrm{mg} / \mathrm{mouse} /$ administration for 5 consecutive days, which was considered the MTD of this administration regimen, did not affect survival, nor did it enhance the efficacy of RIT.

\section{CONCLUSIONS}

In conclusion, when given concurrently with RIT either every 3rd day for a total of 4 administrations or daily for a total of 5 consecutive days, gemcitabine did not enhance the therapeutic efficacy of RIT at the dose regimens employed.

\section{ACKNOWLEDGMENTS}

Part of this study was supported by a grant from the Netherlands Organization for Health Research and Development (ZonMw; Grant number: 920-03-220).

The authors wish to thank Henny Eikholt and Gerry Grutters, Nijmegen, The Netherlands, Central Animal Laboratory (Radboud University Nij- 
megen Medical Center) for their excellent technical assistance in the animal experiments.

\section{REFERENCES}

1. Jain RK. Physiological barriers to delivery of monoclonal antibodies and other macromolecules in tumors. Cancer Res 1990;50:814S.

2. Schlom J, Eggensperger D, Colcher D, et al. Therapeutic advantage of high-affinity anticarcinoma radioimmunoconjugates. Cancer Res 1992;52:1067.

3. Behr TM, Blumenthal RD, Memtsoudis S, et al. Cure of metastatic human colonic cancer in mice with radiolabeled monoclonal antibody fragments. $\underline{\text { Clin Cancer }}$ Res 2000;6:4900.

4. Boerman OC, van Schaijk FG, Oyen WJ, et al. Pretargeted radioimmunotherapy of cancer: Progress step by step. J Nucl Med 2003;44:400.

5. Cardillo TM, Blumenthal R, Ying Z, et al. Combined gemcitabine and radioimmunotherapy for the treatment of pancreatic cancer. Int J Cancer 2002;97:386.

6. Gold DV, Schutsky K, Modrak D, et al. Low-dose radioimmunotherapy ([90]Y-PAM4) combined with gemcitabine for the treatment of experimental pancreatic cancer. Clin Cancer Res 2003;9:3929S.

7. Gold DV, Modrak DE, Schutsky K, et al. Combined ${ }^{90}$ Yttrium-DOTA-labeled PAM4 antibody radioimmunotherapy and gemcitabine radiosensitization for the treatment of a human pancreatic cancer xenograft. Int J Cancer 2004;109:618.

8. Pauwels B, Korst AE, Pattyn GG, et al. Cell-cycle effect of gemcitabine and its role in the radiosensitizing mechanism in vitro. Int J Radiat Oncol Biol Phys 2003;57:1075.

9. Plunkett W, Huang P, Gandhi V. Preclinical characteristics of gemcitabine. Anticancer Drugs 1995;6(Suppl 6):7.

10. McGinn CJ, Shewach DS, Lawrence TS. Radiosensitizing nucleosides. J Natl Cancer Inst 1996;88:1193.

11. Graves SS, Dearstyne E, Lin Y, et al. Combination therapy with pretarget CC49 radioimmunotherapy and gemcitabine prolongs tumor doubling time in a murine xenograft model of colon cancer more effectively than either monotherapy. Clin Cancer Res 2003;9:3712.
12. Koppe MJ, Soede AC, Pels W, et al. Experimental radioimmunotherapy of small peritoneal metastases of colorectal origin. Int J Cancer 2003;106:965.

13. Koppe MJ, Bleichrodt RP, Soede AC, et al. Biodistribution and therapeutic efficacy of (125/131)I-, (186)Re-, (88/90)Y-, or (177)Lu-labeled monoclonal antibody $\mathrm{MN}-14$ to carcinoembryonic antigen in mice with small peritoneal metastases of colorectal origin. $\underline{\mathrm{J} \mathrm{Nucl} \mathrm{Med}}$ 2004;45:1224.

14. Wang H, Li M, Rinehart JJ, et al. Pretreatment with dexamethasone increases antitumor activity of carboplatin and gemcitabine in mice bearing human cancer xenografts: In vivo activity, pharmacokinetics, and clinical implications for cancer chemotherapy. $\underline{\text { Clin Cancer }}$ Res 2004;10:1633.

15. Ey PL, Prowse SJ, Jenkin CR. Isolation of pure IgG1, IgG2a, and IgG2b immunoglobulins from mouse serum using protein A-sepharose. Immunochemistry 1978;15: 429.

16. Lindmo T, Boven E, Cuttitta F, et al. Determination of the immunoreactive fraction of radiolabeled monoclonal antibodies by linear extrapolation to binding at infinite antigen excess. J Immunol Meth 1984;72: 77.

17. Koppe MJ, Oyen WJ, Bleichrodt RP, et al. Combination therapy using the cyclooxygenase- 2 inhibitor parecoxib and radioimmunotherapy in nude mice with small peritoneal metastases of colonic origin. $\underline{\text { Cancer Im- }}$ munol Immunother 2006;55:47.

18. Pauwels B, Korst AE, Lardon F, et al. Combined modality therapy of gemcitabine and radiation. Oncologist 2005;10:34.

19. Mani S, Kugler JW, Knost JA, et al. Phase II trial of 150-minute weekly infusion of gemcitabine in advanced colorectal cancer: Minimal activity in colorectal cancer. Invest New Drugs 1998;16:275.

20. Shewach DS, Hahn TM, Chang E, et al. Metabolism of $2^{\prime}, 2^{\prime}$-difluoro-2'-deoxycytidine and radiation sensitization of human colon carcinoma cells. 1994;54:3218.

21. Shewach DS, Lawrence TS. Radiosensitization of human solid tumor cell lines with gemcitabine. Semin Oncol 1996;23:65.

22. Lawrence TS, Eisbruch A, Shewach DS. Gemcitabinemediated radiosensitization. Semin Oncol 1997;24:S7. 


\section{This article has been cited by:}

1. Yohei Saito, Masahiro Yasunaga, Jun-ichiro Kuroda, Yoshikatsu Koga, Yasuhiro Matsumura. 2010. Antitumour activity of NK012, SN-38-incorporating polymeric micelles, in hypovascular orthotopic pancreatic tumour. European Journal of Cancer 46:3, 650-658. [CrossRef]

2. Zhi-Qiang Huang, Donald J Buchsbaum. 2009. Monoclonal antibodies in the treatment of pancreatic cancer. Immunotherapy 1:2, 223-239. [CrossRef]

3. Andrew J Gunn, Martin W Brechbiel, Peter L Choyke. 2007. The emerging role of molecular imaging and targeted therapeutics in peritoneal carcinomatosis. Expert Opinion on Drug Delivery 4:4, 389-402. [CrossRef] 